REVISTA DE PSIQUIATRIA INFANTO-JUVENIL

ISSN 1130-9512 | E-ISSN 2660-7271

Volumen 38, número 4, octubre-diciembre de 2021, pp. 33-45

Este artículo está bajo licencia Creative Commons

BY-NC-ND 4.0

DOI: $10.31766 /$ revpsij.v38n4a5

\section{Artículo de Revisión}

Recibido: 15/03/21 | Aceptado: 05/11/21

\section{Miguel Ibáñez Alario ${ }^{1 *(1)}$ Soraya Otero Cuesta ${ }^{10}$}

1. Servicio de Psiquiatría y Psicología. Hospital Universitario Marqués de Valdecilla. Santander, España.

*AUTOR DE CORRESPONDENCIA:

Miguel Ibáñez Alario

Correo electrónico: miguel.ibanez@scsalud.es

\section{Farmacogenómica en psiquiatría de niños y adolescentes: Una revisión del estado actual de la cuestión}

\author{
Pharmacogenomics in child and \\ adolescent psychiatry: A review of state- \\ of-the-art status
}

\section{RESUMEN}

La farmacogenómica estudia el rol de los diferentes componentes del genoma humano en la respuesta a fármacos. Conocer la información sobre estos factores podría ser de utilidad en el ámbito de la psicofarmacología de niños y adolescentes para individualizar la selección de tratamientos y minimizar reacciones adversas. Material y métodos: Se ha realizado una búsqueda bibliográfica de publicaciones en los últimos diez años en la base de datos PubMed, utilizando las siguientes palabras clave: Psychiatry AND child and adolescent AND pharmacogenomic/pharmacogenetic. Se seleccionaron 35 artículos siguiendo criterios de inclusión previamente establecidos. Resultados: Los resultados más consistentes son los relativos a los polimorfismos en los genes de la familia CYP450, sobre todo los diferentes fenotipos metabolizadores (lento, intermedio, rápido) que influyen en la respuesta farmacológica de Inhibidores Selectivos de la Recaptación de Serotonina (ISRS), Risperidona y Atomoxetina. Además, los polimorfismos funcionales del gen HTR2A están relacionados con la respuesta clínica de los ISRS y varios polimorfismos de COMT se relacionan con una menor respuesta al Metilfenidato y a otros estimulantes. Variantes de los receptores serotoninérgicos como 5HT2A, 5HT1B, influyen en la respuesta y en la probabilidad de efectos secundarios de los ISRS. Conclusiones: El uso de test farmacogenéticos puede llegar a ser una herramienta complementaria de ayuda en la toma de decisiones terapéuticas en la psiquiatría de niños y adolescentes.

\section{ABSTRACT}

Pharmacogenomics is the science that studies the role of the different components of the human genome on response to drugs. Knowing the information on these factors could be useful in the field of psychopharmacology of children and adolescents in order to individualize the selection of treatments and minimize adverse reactions. Material and methods: A literature review of the publications of the last ten years was carried out in the PubMed database, using the following keywords: Psychiatry AND child and adolescent AND pharmacogenomics; Psychiatry AND child and adolescent AND pharmacogenomic/pharmacogenetic. 35 articles were selected following previously established inclusion criteria. Results: The most consistent results are those related to the polymorphisms of the CYP450 family genes, especially the different metabolising phenotypes (slow, intermediate, fast) that influence pharmacological response to Selective Serotonin Reuptake Inhibitors (SSRIs), Risperidone and Atomoxetine. Furthermore, functional polymorphisms of the HTR2A gene are related to the clinical response to SSRIs and several COMT polymorphisms are related to a decreased response to Methylphenidate and other stimulants. Serotonergic receptor variants such as $5 \mathrm{HT} 2 \mathrm{~A}, 5 \mathrm{HT} 1 \mathrm{~B}$ also influence the response and likelihood of SSRIs' side effects. Conclusions: The use of pharmacogenetic tests may become a complementary tool in therapeutic decision-making in Child and Adolescent Psychiatry. However, in light of the results reviewed, 
No obstante, según los resultados revisados, no

34 está justificado su uso generalizado, aunque podría considerarse en algunos pacientes con enfermedades graves y con respuesta insuficiente a los fármacos ensayados.

Palabras clave: Farmacogenómica, farmacogenética, psicofármacos, psiquiatría del niño y del adolescente.

\section{INTRODUCCIÓN}

La farmacogenómica es la ciencia que estudia el rol de los diferentes componentes del genoma humano en la respuesta a los fármacos (variantes en secuencias genéticas, cambios estructurales en los cromosomas, variantes epigenéticas, variaciones en la expresividad de los genes...), así como al uso de dicha información genómica para individualizar la selección de tratamientos, con el fin de evitar reacciones adversas y potenciar la efectividad.

Existe una gran variabilidad interindividual en la respuesta a los fármacos, así en individuos con un mismo peso y edad, los niveles plasmáticos pueden diferir significativamente y parte de esta variación en la respuesta se debe a factores genéticos que podemos llegar a identificar.

Desde los estudios iniciales sobre la influencia de la genética en las respuestas individuales a los fármacos partiendo de un determinado fenotipo, la ciencia de la farmacogenómica no ha dejado de evolucionar y se ha desarrollado en paralelo a los grandes avances científicos en materia genética, como son el conocimiento del genoma humano, los avances en materia molecular y el proceso de secuenciación del DNA. Así, actualmente, podemos tener mucha más información sobre estos factores que podemos incorporar a la práctica clínica, y que incluso encontramos indexada en los registros de salud informatizados de los hospitales, a la vez que se multiplican los estudios en este campo.

Para mejorar la eficacia de los tratamientos psiquiátricos indicados para niños y adolescentes its widespread use is not justified, although it could be considered in some patients with severe diseases and insufficient response to previously tested drugs.

Keywords:pharmacogenomics, pharmacogenetics, psycotropics, child and adolescent psychiatry.

pudiera ser importante conocer "a priori" la posible respuesta de un paciente a los diversos fármacos con indicación en su patología/s, y por varios motivos, a saber: primero ahorraríamos tiempo y sufrimiento al paciente y a su familia, segundo, se reducirían las prescripciones fallidas, el número de consultas que se generan por este motivo y el gasto farmacéutico, y en tercer lugar, se reduciría la probabilidad de desarrollar efectos adversos indeseados y por tanto todas las consecuencias negativas derivadas de los mismos (1). La identificación de posibles biomarcadores predictores de respuesta, al menos como orientación en la práctica clínica, sería un gran paso para lograr un tratamiento individualizado. Estos biomarcadores, vinculados al metabolismo farmacológico, podrían predecir el efecto de un fármaco según el genotipofenotipo del individuo, optimizando recursos, disminuyendo el tiempo invertido hasta conseguir la respuesta deseada y mejorando la calidad asistencial ofrecida a nuestros pacientes (2).

A destacar la importancia de los diferentes perfiles de metabolizadores en función de la actividad del citocromo $\mathrm{P} 450$, distinguiéndose así varios fenotipos: metabolizadores lentos, intermedios, rápidos y ultrarrápidos. Los metabolizadores lentos tenderán a acumular los diferentes psicofármacos, aumentando los niveles plasmáticos $\mathrm{y}$, por tanto, también los efectos secundarios de los mismos. Al contrario, metabolizadores rápidos o ultrarrápidos no alcanzarían los niveles plasmáticos deseados, esperando encontrar una menor respuesta clínica y menor frecuencia de aparición de secundarismos farmacológicos. 
REVISTA DE PSIQUIATRIA INFANTO-JUVENIL ISSN 1130-9512 | E-ISSN 2660-7271

Volumen 38, número 4, octubre-diciembre de 2021, pp. 33-45

Este artículo está bajo licencia Creative Commons

BY-NC-ND 4.0

Este trabajo tiene como objetivos:

- Conocer los principales biomarcadores farmacogenómicos asociados a los psicofármacos utilizados en psiquiatría de niños y adolescentes.

- Revisar los conocimientos sobre cómo pueden modificar la respuesta a los fármacos con indicación en esta población.

- Revisar los hallazgos sobre su influencia en la aparición de efectos secundarios del uso de los mismos.

\section{MATERIAL Y MÉTODOS}

Se ha realizado una búsqueda bibliográfica de publicaciones en los últimos diez años en la base de datos PubMed, utilizando las siguientes palabras clave: Psychiatry AND child and adolescent AND pharmacogenetic/pharmacogenomic.

La búsqueda se ha limitado a resultados en inglés o en castellano.

La revisión se ha realizado en junio de 2020, encontrándose un total de 102 estudios. Del total de 102 estudios únicamente se seleccionaron 31, los cuales respondían a los tres criterios de inclusión:

- Tener como objetivo estudiar las diferencias en la respuesta a uno o varios psicofármacos.

- Estudio centrado en el ámbito de la psiquiatría.

- Estudio realizado en población infanto-juvenil (niños y adolescentes).

Se han eliminado, por tanto, los estudios centrados en otra temática diferente a la psicofarmacología, los que únicamente son reportes de casos clínicos y los que se han realizado en población adulta, salvo que fuera de especial relevancia para nuestros pacientes.

A estos estudios se han añadido, a través de búsqueda manual, artículos que se han considerado de alta relevancia para complementar la revisión. Al final se han seleccionado un total de 35 artículos (Figura 1).

\section{RESULTADOS}

Se han organizado los resultados en función de las formas en las que los factores genéticos pueden modificar la respuesta a diferentes psicofármacos con el objetivo de distinguir procesos diferentes y aportar claridad.
Farmacogenómica en psiquiatría de niños y adolescentes: Una revisión del estado actual de la cuestión

http://doi.org/10.31766/revpsij.v38n4a5

\section{Alteración en la farmacocinética}

En esta sección se revisan las diferencias que podemos encontrar según variaciones genéticas de las enzimas que intervienen en la absorción, distribución, localización tisular, biotransformación y excreción de los fármacos (Tabla 1).

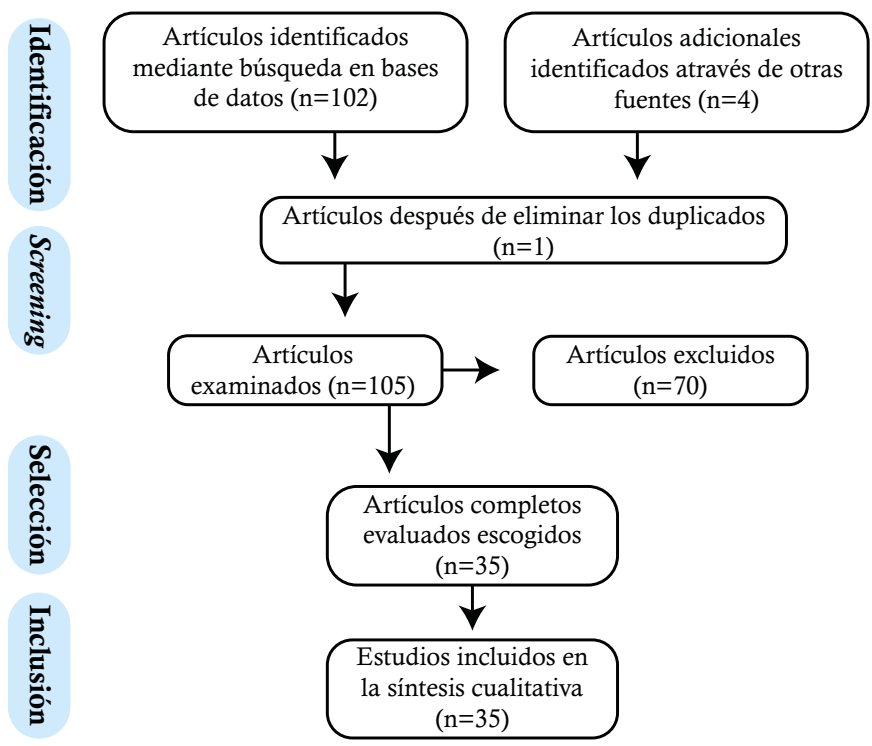

Figura 1. Diagrama de flujos del proceso de selección de artículos para la revisión.

\section{CYP2D6}

E1 CYP2D6 es el citocromo más importante y el que está más implicado en las diferencias en la farmacocinética de varios psicofármacos. Es la enzima P450 mejor caracterizada en humanos y que interviene ampliamente en el metabolismo de infinidad de fármacos. Lo hace, en concreto, mediante reacciones de oxidación de Fase I. CYP2D6 es altamente polimórfico, se conocen alrededor de unas 90 variantes alélicas (3).

Son sustratos principales de dicha enzima, varios inhibidores selectivos de la recaptación de serotonina (ISRS: fluoxetina, paroxetina, fluvoxamina), inhibidores de la recaptación de serotonina y noradrenalina (venlafaxina, duloxetina), inhibidores selectivos de la recaptación de noradrenalina (atomoxetina), moduladores de la actividad serotoninérgica (vortioxetina) y antidepresivos tricíclicos (amitriptilina y nortriptilina). Aunque no todos 
estos fármacos tienen indicación en las poblaciones

de niños y adolescentes, se utilizan fuera de indicación en aquellos casos que no responden a los fármacos habituales. Los pacientes tratados con estos medicamentos y que presenten polimorfismos en esta enzima tendrán cambios en los niveles en plasma de dichos fármacos. Por otro lado, el bupropion (inhibidor de la recaptación de dopamina y noradrenalina) se metaboliza sustancialmente, pero no exclusivamente, por CYP2D6 (4). En el caso de la fluoxetina, diferentes polimorfismos en CYP2D6 y CYP2C9 pueden influir en las concentraciones en sangre de este fármaco (5). Un caso especial es el de la paroxetina, debido a que el propio fármaco puede inhibir este citocromo y su metabolización, realizando un fenómeno de fenoconversión (un individuo con un genotipo de metabolizador rápido puede pasar a ser un metabolizador lento tras el suministro de dicho fármaco).

También hay que señalar que el genotipo de este citocromo varía significativamente entre los diferentes grupos étnicos. La expresión de CYP2D6 está ausente en el 7\% de los caucásicos y afroamericanos, mientras que la deficiencia es rara entre asiáticos (6).

En algunos estudios (7) se ha concluido que anormalidades funcionales en este citocromo pueden estar presentes en más de la mitad de los pacientes de psiquiatría infanto-juvenil.

En un estudio sobre atomoxetina, consistente en administrar una única dosis de dicho fármaco a población infanto-juvenil diagnosticada de Trastorno por déficit de atención con hiperactividad (TDAH), se encontraron diferencias en la concentración plasmática (dosis corregida de fármaco) entre metabolizadores lentos y metabolizadores rápidos (8). Una disminución de la concentración de atomoxetina en metabolizadores rápidos conlleva un menor efecto del fármaco; en cambio, los metabolizadores lentos producen un aumento de la concentración del mismo y efectos secundarios más intensos y frecuentes. Publicada por este mismo equipo en 2019, existe una guía para el genotipado del CYP2D6 previo al inicio de la terapia con atomoxetina (9).

En una revisión realizada en 2018 (10) se analizaron los últimos estudios publicados en población infantojuvenil en relación con variantes farmacogenómicas en el citocromo CYP2D6 y la respuesta clínica / efectos secundarios con el tratamiento con risperidona. Los resultados de los estudios fueron homogéneos en su mayoría, concluyendo que existe una relación entre el perfil metabolizador de dicho citocromo con los niveles de risperidona/9-hidroxi-risperidona en sangre, con aumento de los niveles en los metabolizadores lentos, que presentan más efectos secundarios y disminución de niveles y menor respuesta clínica en los metabolizadores rápidos. Se ha estudiado (11) la influencia del CYP2D6 (concomitantemente con CYP2C9 y el genotipo ABCB1 de la glucoproteína P) en las concentraciones de fluoxetina (y su metabolito principal S-norfluoxetina) en una muestra de 83 niños y adolescentes. La ratio fluoxetina/S-norfluoxetina se correlacionó negativamente con el número de alelos activos de CYP2D6, de tal forma que aquellos individuos con más alelos activos tenían una ratio menor. En aquellos individuos con menores niveles de fluoxetina la respuesta clínica es menor y los individuos con mayores niveles pueden presentar más secundarismos.

\section{CYP2C19}

El CYP2C19 representa entre un $10-20 \%$ de la actividad del citocromo P450 y es el responsable de la metabolización de fármacos como el diazepam, bupropion, tricíclicos (amitriptilina, clomipramina e imipramina) y algunos ISRS como citalopram y escitalopram. La sertralina es metabolizada tanto por el CYP2D6 como por el CYP2C19 aunque su farmacodinámica parece primariamente influenciada por el CYP2C19 (12).

En un estudio retrospectivo (13), publicado en 2019, se publicaron los datos de 263 adolescentes menores de 19 años con trastornos ansioso-depresivos. Se administró citalopram o escitalopram al mismo tiempo que se realizó un genotipado del CYP2C19. Los metabolizadores lentos experimentaron más efectos adversos (hiperactivación, incremento de peso) que los metabolizadores rápidos. Además, hubo más discontinuación en los metabolizadores lentos y los metabolizadores rápidos respondieron de forma precoz al tratamiento. Los resultados, sin embargo, fueron dispares, debido en parte a que la población utilizada tenía diferentes diagnósticos (ansiedad / depresión). 
REVISTA DE PSIQUIATRIA INFANTO-JUVENIL

ISSN 1130-9512 | E-ISSN 2660-7271

Volumen 38, número 4, octubre-diciembre de 2021, pp. 33-45

Este artículo está bajo licencia Creative Commons

BY-NC-ND 4.0
Farmacogenómica en psiquiatría de niños y adolescentes: Una revisión del estado actual de la cuestión

http://doi.org/10.31766/revpsij.v38n4a5

Tabla 1. Psicofármacos, genes-proteínas relacionados, variantes genéticas y su posible implicación en la práctica clínica, derivada de los estudios revisados.

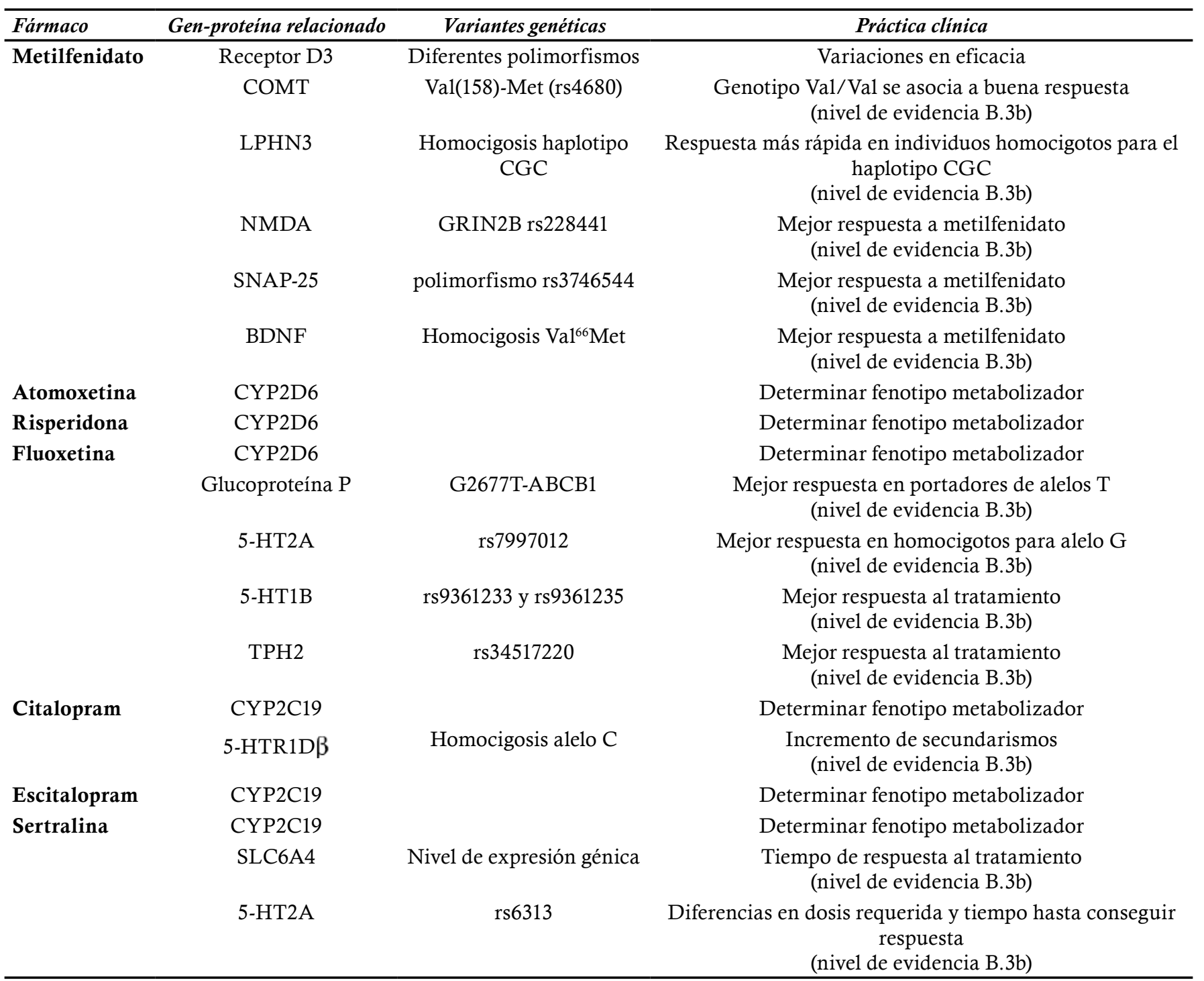

BDNF: factor neurotrófico derivado del cerebro; COMT: Catecol-O-MetilTransferasa ; LPH: latrofilina; NMDA: ácido N-metil-Daspártico; SLC: Solute Carrier Family; SNAP: proteína asociada a sinaptosoma; TPH: triptófano hidroxilasa

Clasificación de niveles de evidencia según Sackett.

A.1a. Revisión sistemática o meta-análisis

A.1b. Ensayos clínicos individuales con intervalo de confianza estrecho

B.2a. Revisión sistemática con homogeneidad de estudio de cohortes.

B.2b. Estudio de cohortes individual / Ensayo clínico de baja calidad

B.3a. Revisión sistemática homogénea de estudio de casos y controles

B.3b. Estudio de casos y controles individuales

C.4. Series de casos. Estudio de casos y controles o cohortes de baja calidad.

D.5. Opiniones de expertos sin evaluación crítica explícita o basada en fisiología o investigación teórica. 
Otro estudio retrospectivo (14) en relación con 38 la administración de sertralina encontró que la dosis máxima durante el periodo inicial de tratamiento se asoció al número de alelos de función reducida de CYP2C19, de tal forma que una disminución en la funcionalidad de dicho citocromo se tradujo en un aumento de la dosis máxima de sertralina conseguida. Sin embargo, el número de alelos de función reducida no se asoció a la dosis a la que los pacientes experimentaron respuesta terapéutica, por lo que la repercusión clínica de dichos resultados es cuestionable. En otro estudio (15) con sertralina y escitalopram los autores concluyeron que la concentración máxima tras la administración del fármaco era mayor en pacientes metabolizadores lentos. Las diferencias fueron más notables en el caso del escitalopram.

\section{Glucoproteína P}

La glucoproteína $\mathrm{P}$ o glucoproteína de permeabilidad se encuentra en la membrana celular y se encarga de expulsar diferentes moléculas al material extracelular. Tiene la función de evitar la acumulación en el sistema nervioso central de muchos psicofármacos. Polimorfismos en el gen de esta proteína se pueden traducir en cambios en su función, aminorando el aclaramiento de muchas de estos fármacos. Se presume que los cambios genómicos puedan estar más relacionados con la proteína final o con la expresión de RNA, ya que ambos se correlacionan mejor con el fenotipo final. E1 polimorfismo G2677T-ABCB1 parece tener cierta influencia en los resultados clínicos obtenidos con el tratamiento con fluoxetina en niños y adolescentes, de tal forma que los portadores de los alelos T podrían tener un mayor grado de mejoría cuando se tratan con dicho fármaco (11).

\section{Alteración en la farmacodinámica}

Los genes que producen una alteración en la farmacodinámica incluyen aquellos encargados de la codificación de transportadores, receptores y enzimas (Tabla 1).

\section{$S L C 6 A 2$}

SLC6A2 (transportador de noradrenalina o solute carrier family 6A2) es el encargado de recaptar noradrenalina desde la hendidura sináptica hasta la neurona presináptica. Asimismo es capaz de devolver dopamina desde el material extracelular. La potencial relevancia de este transportador en patologías como el TDAH ha quedado patente en algunos de los estudios revisados, específicamente el SNP rs3785143 del gen codificador de SLC6A2 parece estar relacionado con los síntomas de inatención (16). En relación con el polimorfismo G1287A de SLC6A2 cabe destacar que los portadores del alelo A podrían tener una mayor variabilidad en el tiempo de respuesta en tareas de atención sostenida (17).

\section{SLC6A3}

SLC6A3 (transportador de dopamina o solute carrier family 6A3) es una proteína de la membrana celular de las neuronas dopaminérgicas. Transporta dopamina desde la hendidura sináptica hacia el interior de la neurona presináptica. La variante SLC6A3 rs2550948 se relaciona con una mayor disminución de sintomatología TDAH tras la administración de metilfenidato en niños con dicha patología (18).

\section{SLC6A4}

E1 SLC6A4 (transportador de serotonina o solute carrier family 6A4) es el encargado de recaptar serotonina desde la hendidura sináptica hasta la neurona presináptica. Es la diana terapéutica de los ISRS. Existen pocos estudios que se centren en las alteraciones farmacodinámicas por variantes genéticas en el gen codificante de SLC6A4, sin embargo, el nivel de expresión de dicho gen podría tener relación con el tiempo de tratamiento con sertralina necesario para alcanzar la remisión en episodios depresivos en niños y adolescentes (14). Así los pacientes con menores niveles de expresión de este gen necesitan más tiempo para conseguir la remisión de los síntomas. 
REVISTA DE PSIQUIATRIA INFANTO-JUVENIL ISSN 1130-9512 | E-ISSN 2660-7271

Volumen 38, número 4, octubre-diciembre de 2021, pp. 33-45

Este artículo está bajo licencia Creative Commons

BY-NC-ND 4.0

\section{Receptor 5-HT2A}

Pertenece a la familia de receptores de serotonina. Es el principal receptor excitatorio de esta familia. El polimorfismo rs6313 se ha relacionado con la dosis de sertralina necesaria para conseguir remisión declínica ansioso-depresiva en población infanto-juvenil, y también con el tiempo de respuesta requerido. Así los pacientes con al menos un alelo A de dicho gen necesitaron dosis máximas del fármaco y tardan más tiempo en responder (14). El polimorfismo rs7997012 se ha relacionado con una mayor mejoría clínica tras introducción de tratamiento antidepresivo con fluoxetina en niños y adolescentes diagnosticados de trastorno depresivo mayor (19). En dicho estudio, los homocigotos para el alelo $G$ fueron los que mayor porcentaje de remisión consiguieron. Otros estudios centrados en la respuesta a escitalopram (disminución de irritabilidad y perseverancia) en pacientes diagnosticados de trastornos del espectro autista no encontraron diferencias entre los dos grupos de diferente genotipo en el gen codificante para 5-HT2A (20).

\section{Receptor 5-HT1B}

Pertenece a la familia de receptores de serotonina. En un estudio (21) se evaluó la influencia de diferentes variantes genéticas relacionadas con este receptor en el tratamiento antidepresivo con fluoxetina en niños y adolescentes diagnosticados de episodio depresivo mayor, encontraron que los polimorfismos rs9361233 y rs9361235 del gen codificante de 5-HT1B se asociaron a una mejor respuesta clínica, en individuos homocigotos. Los heterocigotos para dicho gen mostraron, en cambio, peor respuesta clínica. Otros estudios también han demostrado que variantes genéticas en dicho gen podrían estar relacionadas con respuesta clínica a fluoxetina en esta población (22).

\section{Receptor 5-HTR1D}

Es un componente de la familia de receptores de serotonina. En un estudio centrado en el análisis genético como posible predictor de efectos secundarios
Farmacogenómica en psiquiatría de niños y adolescentes: Una revisión del estado actual de la cuestión http://doi.org/10.31766/revpsij.v38n4a5

en niños con diferentes diagnósticos tratados con citalopram (23) se observó que variaciones genéticas en el gen que codifica dicho receptor (alelo $\mathrm{C}$ en homocigosis) podrían estar asociadas a un incremento en la activación y agitación observada tras varios días de tratamiento. Este hallazgo es relevante, pues estos síntomas se han relacionado con un mayor riesgo de suicidio las primeras semanas post-tratamiento antidepresivo.

\section{Receptor D3}

Componente de la familia de los receptores de dopamina. Codificado por el gen DRD3. Algunos investigadores señalan la importancia de variaciones genéticas en el gen codificante de dicho receptor a la hora de iniciar tratamiento con metilfenidato en niños diagnosticados de TDAH (24), ya que en los individuos con variaciones en dicho gen la ineficacia del tratamiento era hasta 4 veces más probable.

\section{COMT}

La catecol O-metiltransferasa (COMT) es una de las enzimas encargas de la degradación de catecolaminas $\mathrm{y}$, por consiguiente, la inactivación de dichos neurotransmisores en el sistema nervioso central, donde se encuentra en el espacio intracelular neuronal. El polimorfismo Val(158)-Met (rs4680) ha demostrado ser potencialmente relevante en el tratamiento con metilfenidato, de tal forma que se ha encontrado asociación (estadísticamente no significativa) entre el genotipo Val/Val del gen codificante de la COMT y una buena respuesta a dicho tratamiento (disminución de la impulsividad y la hiperactividad tras el inicio de metilfenidato) (25).

\section{LPHN3}

LPHN3 o latrofilina 3 forma parte de la familia de los receptores asociados a proteína $G$, específico del cerebro. Se encarga de la adhesión celular y la transmisión de señal. Se ha sugerido que el haplotipo CGC del cromosoma 11 está asociado con la susceptibilidad genética a padecer TDAH y que hay una asociación entre los individuos homocigotos 
para el haplotipo CGC y una respuesta más rápida al

\section{NMDA}

El NMDA (ácido N-metil-D-aspártico) es un derivado aminoácido que actúa como un agonista específico en el receptor NMDA. Tiene similar acción al neurotransmisor glutamato. El genotipo GRIN2B rs228441 podría ser un buen predictor de respuesta al tratamiento con metilfenidato en niños diagnosticados de TDAH, debido a que se ha hallado una asociación entre dicha variante genética y una mejor respuesta a dicho fármaco (disminución de inatención, hiperactividad e impulsividad) (27).

\section{SNAP-25}

La proteína asociada a sinaptosoma $25 \mathrm{kDa}$ (SNAP-25) es la encargada de facilitar la fusión de vesículas desde el material extracelular con los compartimentos intracelulares. Concretamente regula la capacidad de respuesta sináptica al calcio a través de la unión de las vesículas con la membrana extracelular. El polimorfismo rs3746544 parece estar asociado a una mejor respuesta a metilfenidato en niños diagnosticados de TDAH (17). Además, en otro estudio sobre el gen codificante de dicha proteína, se encontró que aquellos niños (con diagnóstico de TDAH) con, al menos, un alelo $\mathrm{T}$ en el gen de SNAP25 (polimorfismo $1065 \mathrm{~T}>\mathrm{G}$ ) tenían una mejor respuesta a metilfenidato (28).

\section{$B D N F$}

El BDNF (factor neurotrófico derivado del cerebro) es una proteína de la familia de las neurotrofinas que actúa como factor de crecimiento. Está asociado a los procesos de plasticidad, desarrollo y supervivencia del sistema nervioso. Un estudio (29) sobre la relación entre las variantes del gen codificante de BDNF con la respuesta a metilfenidato en población infantil diagnosticada de TDAH, encontró que los pacientes homocigotos para el polimorfismo $\mathrm{Val}^{66} \mathrm{Met}$ tenían una mejor respuesta clínica al fármaco.

\section{TPH2}

La TPH2 (triptófano hidroxilasa 2) es una enzima relacionada con la síntesis de serotonina, que se expresa sobre todo en las neuronas serotoninérgicas (núcleos del rafe). Un estudio (30) encontró que la presencia de tres polimorfismos (rs11179002, rs60032326 y rs34517220) en los lugares de unión de factores de transcripción del gen codificador de TPH2 se asociaba significativamente con una mejor respuesta al tratamiento con fluoxetina en población infanto-juvenil diagnosticada de trastorno depresivo. Los resultados fueron de mayor magnitud con el polimorfismo rs34517220.

\section{Otros}

Cabe destacar que la FDA recomienda testar HLA-B1502 previo uso de carbamazepina, debido a que los portadores de una mutación en dicho gen tienen un riesgo estimado de desarrollar síndrome de Stevens-Johnson diez veces mayor (31).

Se ha revisado otro estudio en el que ciertas variantes genéticas se asocian con diferencias en el incremento de índice de masa corporal en niños $\mathrm{y}$ adolescentes que comienzan tratamiento con risperidona (32). La variante genética rs1137101 del receptor de leptina (LEPR) está directamente relacionada con el incremento de peso durante el tratamiento con dicho fármaco.

\section{DISCUSIÓN}

Teniendo en cuenta la información revisada, parece evidente el papel prometedor que puede llegar a jugar la farmacogenómica en la elección del tratamiento óptimo e individualizado para cada paciente en las consultas de nuestra especialidad. Los test farmacogenéticos además serían de gran utilidad a la hora de complementar las guías clínicas de dosificación y monitorización de eficacia y de posibles efectos secundarios. En la actualidad la toma de decisiones sobre la indicación de fármacos se basa de forma casi exclusiva en la medicina basada en la evidencia y en las recomendaciones de las guías clínicas, pero no nos permiten saber de antemano 
REVISTA DE PSIQUIATRIA INFANTO-JUVENIL ISSN 1130-9512 | E-ISSN 2660-7271

Volumen 38, número 4, octubre-diciembre de 2021, pp. 33-45

Este artículo está bajo licencia Creative Commons

BY-NC-ND 4.0

la eficacia y los posibles efectos secundarios que un fármaco o combinación de varios fármacos puede tener en un/a paciente concreto/a.

Aunque los avances en este campo van parejos al desarrollo exponencial de la genómica, los resultados de los estudios son aún poco consistentes, las muestras son muy heterogéneas (33) y la replicación de los hallazgos es aún escasa, en algunos casos inexistente.

Nuestra revisión se ha limitado a los estudios publicados en la última década, y en muestras de niños y adolescentes, pero es posible que los resultados encontrados en poblaciones adultas sean extrapolables a nuestras poblaciones. Así por ejemplo el aripiprazol es un fármaco con indicaciones múltiples en niños y adolescentes, y cuenta con recomendaciones específicas de la FDA sobre su uso, aconsejando dosis más bajas en pacientes con fenotipo CYP2D6 de metabolizadores lentos (34). De nuestra revisión podemos afirmar que algunos hallazgos son más consistentes y son los que cuentan con más opciones de ser incluidos en test genómicos con mayor validez (véase tabla 1 )

Así, los polimorfismos en los genes de la familia CYP450, sobre todo los diferentes fenotipos metabolizadores (lento, intermedio, rápido) de CYP2D6, CYP2C19 y CYP3A4, influyen en la respuesta farmacológica de múltiples fármacos como los ISRS, risperidona o atomoxetina, que son ampliamente utilizados en poblaciones de niños y adolescentes.

Asimismo, se han identificado múltiples polimorfismos funcionales del gen HTR2A que están relacionados con la respuesta clínica de los ISRS y varios polimorfismos de COMT que se relacionan con una menor respuesta al metilfenidato y a otros estimulantes y por tanto menos eficacia en el tratamiento del TDAH de estos pacientes.

Variantes de los receptores serotoninérgicos como 5HT2A, 5HT1B, influyen también en la respuesta y en la probabilidad de efectos secundarios de los ISRS (fluoxetina y sertralina) más utilizados en nuestras poblaciones.

La farmacogenómica es una ciencia joven, con un desarrollo rápido, y con un crecimiento exponencial en el número de publicaciones, la mayoría realizadas en población de adultos. A pesar de este gran
Farmacogenómica en psiquiatría de niños y adolescentes: Una revisión del estado actual de la cuestión http://doi.org/10.31766/revpsij.v38n4a5

desarrollo, todavía no hay identificados marcadores genéticos clínicamente significativos, aunque es de esperar que se produzcan cambios a corto plazo en este sentido. De cara a decidir implementar test de farmacogenómica en la práctica clínica, sería necesario tener más datos que nos pudieran ayudar en la toma de decisiones, conociendo de antemano qué fármacos tienen mayor riesgo de provocar efectos secundarios y/o de ser menos eficaces en el tratamiento de la patología de nuestros pacientes. Por otro lado, sería necesario incluir en las bases de datos otros factores como el estilo de vida (alimentación, deporte, toma de suplementos alimenticios), y consumo de tóxicos (tabaco, cannabis, alcohol) en el caso de los adolescentes, que puedan modificar la respuesta de un paciente a un determinado fármaco.

La totalidad de los test farmacogenómicos disponibles en la actualidad están comercializados por empresas privadas y, aunque presentan una adecuada validez clínica, al no haber una estandarización en los genes o en las variantes alélicas que contienen estos test los resultados pueden diferir entre laboratorios, lo que añade más confusión a la hora de interpretar la utilidad de los resultados en la práctica clínica. (35)

Aunque en nuestra revisión no hemos encontrado estudios de coste/beneficio sobre el uso de estas pruebas en población de niños y adolescentes, podemos sugerir que las indicaciones de realizar test farmacogenéticos en las que el coste/beneficio "a priori" podría ser más favorable serían las siguientes:

- Pacientes con patologías graves, ya que al mejorar la precisión de la indicación se reducirían los tiempos de ingreso, la frecuencia de consultas, los efectos secundarios, así como otros gastos indirectos como los costes por traslados, perdidas laborales para los cuidadores y de escolarización para el paciente.

- Pacientes con historia de fracasos terapéuticos y/o incremento de probabilidad de efectos secundarios graves de algunos grupos de medicamentos ensayados.

- Pacientes polimedicados y/o con múltiples patologías en los que precisar más las indicaciones podría reducir el riesgo de interacciones medicamentosas y de los efectos de la toxicidad de las asociaciones de fármacos. 
A medida que se incrementen los estudios 42 aleatorizados y doble ciego, así como la replicación de resultados, y se reduzcan los costes de estas pruebas, será posible utilizar los test farmacogenómicos como una herramienta complementaria de indudable utilidad para orientar la elección de un fármaco o fármacos para un paciente concreto. En este sentido es indispensable que, para clarificar la utilidad de estas pruebas, se realicen además estudios fármacoeconómicos de coste-beneficio que evalúen la viabilidad de su implementación en la práctica clínica diaria. Pese a los costes que pueda acarrear la realización de dichas pruebas, el beneficio potencial de mejorar los resultados en la salud mental de los niños y

adolescentes es considerable. Además, como hemos visto, la realización de estas pruebas podría reducir los costes al optimizar la selección de tratamientos y disminuir otros procedimientos que llevan consigo más gasto (hospitalizaciones, ensayo de múltiples fármacos, polifarmacia, respuesta ante necesidades especiales etc.).

Aunque nuestra revisión no avala la utilidad generalizada de los test farmacogenéticos en la práctica clínica en el momento actual, las determinaciones de HLA-A y HLA-B serían recomendables antes de decidir usar carbamazepina y/o oxcarbamazepina (31). En caso de estar disponibles, en aquellos pacientes con historia de respuesta insuficiente o de efectos secundarios, sería recomendable realizar las determinaciones de CYP2C19 y CYP2D6, ya que los pacientes con fenotipos de metabolismo alterado son de mayor riesgo para efectos secundarios y posiblemente menos respondedores para medicamentos del grupo de antidepresivos y de antipsicóticos (35).

\section{CONCLUSIONES}

La farmacogenética se ofrece como un campo prometedor y en rápido desarrollo y es probable que llegue a convertirse, en un futuro próximo, en una herramienta complementaria de utilidad en la toma de decisiones clínicas en el ámbito de la psiquiatría, y también en el de nuestra población.

Es necesario que se realicen más estudios con una adecuada metodología y con replicación de los hallazgos, así como reducir la heterogeneidad de las muestras.

La revisión de las publicaciones en población de niños y adolescentes, hasta la fecha, no justifica el uso generalizado de los test farmacogenéticos, aunque podrían ser considerados con precaución en algunos casos concretos sobre todo si se identifica la posibilidad de efectos secundarios de gravedad, o en pacientes con enfermedades graves y/o con respuesta insuficiente a los fármacos ensayados.

\section{CONFLICTO DE INTERESES}

Los autores no declaran ningún conflicto de intereses.

\section{REFERENCIAS}

1. Turner RM. From the lab to the prescription pad: genetics, CYP450 analysis, and medication response. J Child Adolesc Psychiatr Nurs. 2013 May;26(2):119-23.

https://doi.org/10.1111/jcap.12028

2. Wall CA, Croarkin PE, Swintak C, Koplin BA. Psychiatric pharmacogenomics in pediatric psychopharmacology. Child Adolesc Psychiatr Clin N Am. 2012 Oct;21(4):773-88.

https://doi.org/10.1016/j.chc.2012.07.001

3. Wehry AM, Ramsey L, Dulemba SE, Mossman SA, Strawn JR. Pharmacogenomic Testing in Child and Adolescent Psychiatry: An EvidenceBased Review. Curr Probl Pediatr Adolesc Health Care. 2018 Feb;48(2):40-49.

https://doi.org/10.1016/j.cppeds.2017.12.003

4. Namerow LB, Walker SA, Loftus M, Bishop JR, Ruaño G, Malik S. Pharmacogenomics: An Update for Child and Adolescent Psychiatry. Curr Psychiatry Rep. 2020 May 6;22(5):26.

https://doi.org/10.1007/s11920-020-01145-4. PMID: 32377970.

5. Blazquez A, Mas S, Plana MT, Lafuente A, Lázaro L. Fluoxetine pharmacogenetics in child and adult populations. Eur Child Adolesc Psychiatry. 2012 Nov;21(11):599-610.

https://doi.org/10.1007/s00787-012-0305-6 
REVISTA DE PSIQUIATRIA INFANTO-JUVENIL

ISSN 1130-9512 | E-ISSN 2660-7271

Volumen 38, número 4, octubre-diciembre de 2021, pp. 33-45

Este artículo está bajo licencia Creative Commons

BY-NC-ND 4.0

6. Mizutani T. PM frequencies of major CYPs in Asians and Caucasians. Drug Metab Rev. 2003;35(2-3):99-106.

https://doi.org/10.1081/dmr-120023681.

7. Thümmler S, Dor E, David R, Leali G, Battista M, David A, Askenazy F, Verstuyft C. Pharmacoresistant Severe Mental Health Disorders in Children and Adolescents: Functional Abnormalities of Cytochrome P450 2D6. Front Psychiatry. 2018 Jan 24;9:2.

https://doi.org/10.3389/fpsyt.2018.00002

8. Brown JT, Abdel-Rahman SM, van Haandel L, Gaedigk A, Lin YS, Leeder JS. Single dose, CYP2D6 genotype-stratified pharmacokinetic study of atomoxetine in children with ADHD. Clin Pharmacol Ther. 2016;99(6):642-50.

https://doi.org/10.1002/cpt.319

9. Brown JT, Bishop JR, Sangkuhl K, Nurmi EL, Mueller DJ, Dinh JC, Gaedigk A, Klein TE, Caudle KE, McCracken JT, De Leon J, Leeder JS. Clinical Pharmacogenetics Implementation Consortium Guideline for Cytochrome P450 (CYP)2D6 Genotype and Atomoxetine Therapy. Clin Pharmacol Ther. 2019 Jul;106(1):94-102. https://doi.org/10.1002/cpt.1409

10. Dodsworth T, Kim DD, Procyshyn RM, Ross CJ, Honer WG, Barr AM. A systematic review of the effects of CYP2D6 phenotypes on risperidone treatment in children and adolescents. Child Adolesc Psychiatry Ment Health. 2018 Jul 16;12:37.

\section{https://doi.org/10.1186/s13034-018-0243-2}

11. GassóP,RodríguezN,Mas S,PagerolsM, Blázquez A, Plana MT, Torra M, Lázaro L, Lafuente A. Effect of CYP2D6, CYP2C9 and ABCB1 genotypes on fluoxetine plasma concentrations and clinical improvement in children and adolescent patients. Pharmacogenomics J. 2014 Oct;14(5):457-62.

https://doi.org/10.1038/tpj.2014.12

12. Ramsey LB, Bishop JR, Strawn JR. Pharmacogenetics of treating pediatric anxiety and depression. Pharmacogenomics. 2019 Aug;20(12):867-70. https:// doi.org/10.2217/pgs-2019-0088

13. Aldrich SL, Poweleit EA, Prows CA, Martin LJ, Strawn JR, Ramsey LB. Influence of CYP2C19 Metabolizer Status on Escitalopram/Citalopram
Farmacogenómica en psiquiatría de niños y adolescentes: Una revisión del estado actual de la cuestión

http://doi.org/10.31766/revpsij.v38n4a5

Tolerability and Response in Youth With Anxiety and Depressive Disorders. Front Pharmacol. 2019 Feb 19;10:99.

https://doi.org/10.3389/fphar.2019.00099

14. Poweleit EA, Aldrich SL, Martin LJ, Hahn D, Strawn JR, Ramsey LB. Pharmacogenetics of Sertraline Tolerability and Response in Pediatric Anxiety and Depressive Disorders. J Child Adolesc Psychopharmacol. 2019 Jun;29(5):34861. https://doi.org/10.1089/cap.2019.0017

15. Strawn JR, Poweleit EA, Ramsey LB. CYP2C19Guided Escitalopram and Sertraline Dosing in Pediatric Patients: A Pharmacokinetic Modeling Study. J Child Adolesc Psychopharmacol. 2019 Jun;29(5):340-47.

https://doi.org/10.1089/cap.2018.0160

16. Angyal N, Horvath EZ, Tarnok Z, Richman MJ, Bognar E, Lakatos K, Sasvari-Szekely M, Nemoda Z. Association analysis of norepinephrine transporter polymorphisms and methylphenidate response in ADHD patients. Prog Neuropsychopharmacol Biol Psychiatry. 2018 Jun 8;84(Pt A):122-28.

https://doi.org/10.1016/j.pnpbp.2018.01.013

17. Kim BN, Kim JW, Cummins TD, Bellgrove MA, Hawi Z, Hong SB, Yang YH, Kim HJ, Shin MS, Cho SC, Kim JH, Son JW, Shin YM, Chung US, Han DH. Norepinephrine genes predict response time variability and methylphenidateinduced changes in neuropsychological function in attention deficit hyperactivity disorder. J Clin Psychopharmacol. 2013 Jun;33(3):356-62. https://doi.org/10.1097/JCP.0b013e31828f9fc3

18. Gomez-Sanchez CI, Carballo JJ, Riveiro-Alvarez R, Soto-Insuga V, Rodrigo M, MahilloFernandez I, Abad-Santos F, Dal-Ré R, Ayuso C. Pharmacogenetics of methylphenidate in childhood attention-deficit/hyperactivity disorder: long-term effects. Sci Rep. 2017 Sep 4;7(1):10391. https://doi.org/10.1038/s41598-017-10912-y

19. Gassó P, Blázquez A, Rodríguez N, Boloc D, Torres T, Mas S, Lafuente A, Lázaro L. Further Support for the Involvement of Genetic Variants Related to the Serotonergic Pathway in the Antidepressant Response in Children and Adolescents After a 12-Month Follow-Up: Impact of the HTR2A rs7997012 Polymorphism. J Child 
Adolesc Psychopharmacol. 2018 Dec;28(10):711718. https://doi.org/10.1089/cap.2018.0004

20. Najjar F, Owley T, Mosconi MW, Jacob S, Hur K, Guter SJ, Sweeney JA, Gibbons RD, Cook EH, Bishop JR. Pharmacogenetic Study of Serotonin Transporter and 5HT2A Genotypes in Autism. J Child Adolesc Psychopharmacol. 2015 Aug;25(6):467-74. https://doi.org/10.1089/cap.2014.0158. PMID: 26262902; PMCID: PMC4544820.

21. Gassó P, Rodríguez N, Blázquez A, Monteagudo A, Boloc D, Plana MT, Lafuente A, Lázaro L, Arnaiz JA, Mas S. Epigenetic and genetic variants in the HTR1B gene and clinical improvement in children and adolescents treated with fluoxetine. Prog Neuropsychopharmacol Biol Psychiatry. 2017 Apr 3;75:28-34.

https://doi.org/10.1016/j.pnpbp.2016.12.003

22. Mas S, Blázquez A, Rodríguez N, Boloc D, Lafuente A, Arnaiz JA, Lázaro L, Gassó P. Pharmacogenetic study focused on fluoxetine pharmacodyna-mics in children and adolescent patients: impact of the serotonin pathway. Pharmacogenet Genomics. 2016 Nov;26(11):487-96. https://doi.org/10.1097/ FPC.0000000000000240

23. Amitai M, Kronenberg S, Carmel M, Michaelovsky E, Frisch A, Brent D, Apter A, Chen A, Weizman A, Fennig S. Pharmacogenetics of citalopram-related side effects in children with depression and/or anxiety disorders. J Neural Transm (Vienna). 2016 Nov;123(11):1347-1354. https://doi.org/10.1007/s00702-016-1585-7

24. Pagerols M, Richarte V, Sánchez-Mora C, GarciaMartínez I, Corrales M, Corominas M, Cormand B, Casas M, Ribasés M, Ramos-Quiroga JA. Pharmacogenetics of methylphenidate response and tolerability in attention-deficit/hyperactivity disorder. Pharmacogenomics J. 2017 Jan;17(1):98104. https://doi.org/10.1038/tpj.2015.89

25. ParkS, Kim JW, Kim BN, Shin MS, YooHJ, ChoSC. Catechol-O-methyltransferase Val158-Met polymorphism and a response of hyperactive-impulsive symptoms to methylphenidate: A replication study from South Korea. J Psychopharmacol. 2014 Jul;28(7):671-6.

https://doi.org/10.1177/0269881114527654
26. Bruxel EM, Salatino-Oliveira A, AkutagavaMartins GC, Tovo-Rodrigues L, Genro JP, Zeni CP, Polanczyk GV, Chazan R, Schmitz M, Arcos-Burgos M, Rohde LA, Hutz MH. LPHN3 and attention-deficit/hyperactivity disorder: a susceptibility and pharmacogenetic study. Genes Brain Behav. 2015 Jun;14(5):419-27. https://doi.org/10.1111/gbb.12224

27. Kim JI, Kim JW, Park JE, Park S, Hong SB, Han DH, Cheong JH, Choi JW, Lee S, Kim BN. Association of the GRIN2B rs2284411 polymorphism with methylphenidate response in attention-deficit/hyperactivity disorder. J Psychopharmacol. 2017 Aug;31(8):1070-77. https://doi.org/10.1177/0269881116667707

28. Song J, Kim SW, Hong HJ, Lee MG, Lee BW, Choi TK, Lee SH, Yook KH. Association of SNAP-25, SLC6A2, and LPHN3 with OROS methylphenidate treatment response in attention-deficit/ hyperactivity disorder. Clin Neuropharmacol. 2014 Sep-Oct;37(5):136-41. https://doi.org/10.1097/ WNF.0000000000000045

29. Kim BN, Cummins TD, Kim JW, Bellgrove MA, Hong SB, Song SH, Shin MS, Cho SC, Kim JH, Son JW, Shin YM, Chung US, Han DH. Val/Val genotype of brain-derived neurotrophic factor (BDNF) $\mathrm{Val}^{66} \mathrm{Met}$ polymorphism is associated with a better response to OROS-MPH in Korean ADHD children. Int J Neuropsychopharmacol. 2011 Nov;14(10):1399-410. https://doi.org/10.1017/S146114571100099X

30. Gassó P, Rodríguez N, Boloc D, Blázquez A, Torres T, Gortat A, Plana MT, Lafuente A, Mas S, Arnaiz JA, Lázaro L. Association of regulatory $\mathrm{TPH} 2$ polymorphisms with higher reduction in depressive symptoms in children and adolescents treated with fluoxetine. Prog Neuropsychopharmacol Biol Psychiatry. 2017 Jul 3;77:236-40.

https://doi.org/10.1016/j.pnpbp.2017.04.031

31. Phillips EJ, Sukasem C, Whirl-Carrillo M, Müller DJ, Dunnenberger HM, Chantratita W, Goldspiel B, Chen YT, Carleton BC, George AL Jr., Mushiroda T, Klein T, Gammal RS, Pirmohamed M. Clinical pharmacogenetics implementation consortium guideline for HLA genotype and use of carbamazepine and oxcarbazepine: 2017 
REVISTA DE PSIQUIATRIA INFANTO-JUVENIL ISSN 1130-9512 | E-ISSN 2660-7271

Volumen 38, número 4, octubre-diciembre de 2021, pp. 33-45

Este artículo está bajo licencia Creative Commons

BY-NC-ND 4.0

update. Clin. Pharmacol. Ther. 2018;103, 574-81. https://doi.org/10.1002/cpt.1004

32. Almandil NB, Lodhi RJ, Ren H, Besag FMC, Rossolatos D, Ohlsen R, Slomp C, Lapetina DL, Plazzotta G, Murray ML, Al-Sulaiman AA, Gringras P, Wong ICK, Aitchison KJ. Associations between the LEP -2548G/A Promoter and Baseline Weight and between LEPR Gln223Arg and Lys656Asn Variants and Change in BMI z Scores in Arab Children and Adolescents Treated with Risperidone. Mol Neuropsychiatry. 2018 Oct;4(2):111-17.

https://doi.org/10.1159/000490463

33. Maruf AA, Greenslade A, Arnold PD, Bousman C. Antidepressant pharmacogenetics in children
Farmacogenómica en psiquiatría de niños y adolescentes: Una revisión del estado actual de la cuestión

http://doi.org/10.31766/revpsij.v38n4a5

and young adults: A systematic review. J Affect Disord. 2019 Jul 1;254: 98-108.

34. Dean L. Aripiprazole Therapy and CYP2D6 Genotype. 2016 Sep 22. In: Pratt VM, McLeod HL, Rubinstein WS, et al., editors. Medical Genetics Summaries [Internet]. Bethesda (MD): National Center for Biotechnology Information (US); 2012. https://www.ncbi.nlm.nih.gov/books/ NBK385288/.

35. McMahon F, Degenhardt F. Genetic Testing and Psychiatric Disorders: A Statement from the International Society of Psychiatric Genetics (Updated and approved 3/11/2019).

https://ispg.net/genetic-testing-statement/ 